\title{
¿Qué teorías sobre el aprendizaje de la lectura se atribuyen los profesores que enseñan a leer con diferente metodología?
}

\author{
Natalia Suárez ${ }^{1}$, Cristina Rodríguez ${ }^{2}$, Isabel O’Shanahan ${ }^{1}$ y Juan E. Jiménez ${ }^{1}$ \\ ${ }^{1}$ Universidad de La Laguna (España); ${ }^{2}$ Universidad de Amsterdam (Países Bajos)
}

El objetivo de este estudio ha sido analizar cuáles son las teorías sobre el aprendizaje de la lectura que se atribuye el profesorado de educación infantil y educación primaria según la metodología de enseñanza de la lectura que dicen utilizar. Para ello, contamos con una muestra total de 522 docentes que cumplimentaron un cuestionario atribucional que forma parte del programa tutorial LETRA (www.programaletra.ull.es) que está orientado a la formación del profesorado tutor y de apoyo para la enseñanza de la lectura. Los resultados nos muestran que los profesores que se atribuyen teorías sobre el aprendizaje de la lectura con una orientación sociocultural se inclinan más por un método mixto; los profesores con una orientación más conductista piensan que la repetición en sus ejercicios y en la instrucción es importante para enseñar a leer, y se inclinan más por la utilización de un método silábico y mixto. Por último, los resultados nos indican que los profesores que se atribuyen una teoría constructivista del aprendizaje se inclinan más por la utilización de métodos globales y mixtos para enseñar a leer a sus alumnos.

Palabras clave: Creencias, teorías implícitas, teorías del aprendizaje, programa Letra, métodos de enseñanza de lectura.

What learning theories of reading are attributed by teachers who teach reading with different teaching method? The aim of this study was to analyze the teacher's theories about learning to read and reading instruction that claim to use in the classroom. To do this, we selected a total sample of 522 kindergarden and primary teachers in service who completed an attributional questionnaire across the tutorial program LETRA (www.programaletra.ull.es). The results showed that teachers attributed theories of learning to read with a sociocultural orientation lean more towards a mixed method, teachers who share a behavior approach consider that the repetition in your workouts and instruction is important to teach reading, are more inclined to use a syllabic method and mixed. Moreover, the results indicate that teachers who hold constructivist theory they select whole-language and mixed approaches for teaching reading to their students.

Keywords: Teacher's beliefs, implicit theories, learning theories, Letra program, reading instruction.

Correspondencia: Natalia Suárez. Departamento de Psicología Evolutiva y de la Educación. Facultad de Psicología, Universidad de la Laguna. Campus de Guajara, s/n. Despacho B3-02. C.P.: 38200. La Laguna, Tenerife (España). E-mail: nsuaru@ull.es 
El estudio del pensamiento del profesor se ha enfocado desde múltiples perspectivas: creencias, teorías implícitas y modelos. Las creencias son las verdades personales indiscutibles sustentadas por cada uno, derivadas de la experiencia o de la fantasía, que tienen un fuerte componente evaluativo, afectivo y se manifiestan a través de declaraciones verbales o de acciones (Pajares, 1992). Cuando hablamos de teorías implícitas nos referimos a un grupo de principios que están conectados en sistemas de creencias, normalmente apoyadas por una investigación extensa. Las teorías implícitas son teorías pedagógicas personales reconstruidas sobre la base de conocimientos académicos históricamente elaborados y transmitidos a través de la formación. Rodrigo (1993) llegó a proponer la existencia de dos niveles funcionales en las teorías: síntesis de conocimiento y síntesis de creencias. Por un lado, una teoría opera al nivel de conocimiento cuando la persona utiliza la teoría de forma declarativa para reconocer o discriminar entre varias ideas, producir expresiones verbales sobre el dominio de la teoría. Por otro lado, el nivel de creencias se desencadena cuando las personas utilizan la teoría de modo pragmático para interpretar situaciones, realizar inferencias prácticas para la comprensión y predicción de sucesos, así como planificar la conducta. La distinción entre ambos niveles se establece en función de que la demanda tenga una orientación teórica o pragmática.

Durante casi tres décadas, la investigación ha documentado la influencia de las creencias de los profesores sobre la práctica educativa (Clark y Peterson, 1986; Dubois, 2002; Granda, 1998; Hayes, 1997; Shavelson y Stern, 1981). Incluso se ha sugerido que si los docentes son conscientes de sus propias creencias, el repertorio de habilidades de enseñanza puede verse modificado provocando un cambio en la toma de decisiones en el aula y en las estrategias de enseñanza y evaluación (Tracey y Mandel, 2012). Esta aportación nos demuestra que si queremos lograr cambios en la enseñanza es necesario adentrarse en las concepciones del profesorado y modificarlas (McAlpine y Weston, 2002). Así, por ejemplo, Kuzborska (2011) trató de averiguar cuáles serían las creencias de los docentes sobre la enseñanza de la lectura y su relación con las prácticas en el aula. Los resultados mostraron que los docentes piensan que las habilidades que mejoran el desarrollo lector son: el vocabulario, la comprensión, la lectura en voz alta, la discusión de los textos en clase, el aprendizaje colaborativo, enseñar a resolver los problemas de comprensión, leer con una propuesta y tener un monitor de lectura que oriente al alumnado. Además, se llevaron a cabo grabaciones a los profesores mientras enseñaban a leer a sus alumnos y encontraron que sus creencias fueron congruentes con sus prácticas.

En este sentido, muchas investigaciones han demostrado que los eventos instruccionales pueden ser catalizadores del cambio de creencias (Fazio, 2000, 2003; Matanzo y Harris, 1999; Stevens, 2002; Theurer, 2002; Wolf et al., 1996) ya que las creencias son estructuras mentales permeables, susceptibles de ser modificadas 
(Thompson, 1992), a pesar de que no exista hoy en día consenso en ello (Block y Hazelip, 1995; Richardson ,1996).

Se han llegado a formular distintas teorías sobre el aprendizaje de la lectura: innatista, maduracionista, conductista, sociocultural, constructivista y psicolingüística.

Desde la teoría maduracionista se ha postulado que los niños necesitan madurar y desarrollar su conocimiento antes de comenzar el proceso de aprendizaje de la lectura.

La teoría conductista postula que la conducta es aprendida a través de las propias experiencias del individuo, por lo que la adquisición de cualquier tipo de conducta, en este caso de la lectura, se encuentra influida por las características del medio-ambiente.

Frente a la teoría conductista nos encontramos en otro extremo a la teoría sociocultural que viene a postular que las funciones mentales superiores parten de la vida social y para comprender al individuo es necesario comprender las relaciones sociales del mismo (Vigotsky, 1979). Sin embargo, por otro lado, el constructivismo, postula que el proceso de construcción del conocimiento es un proceso fundamentalmente interno e individual, basado en el proceso de equilibración, que la influencia del medio sólo puede favorecer o dificultar.

La teoría innatista formula que el ser humano nace con predisposición para aprender y, en este caso, la premisa básica que se deriva de este enfoque es que sería posible el aprendizaje precoz de la lectura. Por último, la teoría psicolingüística considera que la lectura es una actividad compleja y múltiple, en donde se hace necesario coordinar una serie de procesos automáticos y no conscientes (ver Tracey y Mandel, 2012, para una descripción exhaustiva de las teorías). Muchas de estas teorías pueden ser compartidas por los docentes que enseñan a leer. En este sentido, nos planteamos en el presente estudio averiguar qué teorías del aprendizaje se atribuyen los profesores que enseñan a leer con diferente metodología.

\section{Enseñanza de la lectura}

Los métodos sintéticos parten de la unidad más pequeña a la más compleja, es decir, parten de lo más abstracto para llegar a lo concreto. Habitualmente, se aprenden las vocales, para introducir poco a poco las diferentes consonantes. Además, es normal que se aprenda a escribir la letra a la vez que su lectura. Estos métodos pueden ser: fonéticos: se aprenden las letras por su sonido, es decir, se aprende la "m" de "miau", la "s" de "sssssss". Incluso, los docentes utilizan onomatopeyas para relacionar los sonidos de las letras con los sonidos que realizan, por ejemplo, los animales. Las letras se van combinando poco a poco, a medida que el niño las va conociendo. El profesor lleva a cabo actividades centradas en la enseñanza del sonido de las letras del abecedario, se apoya en ilustraciones y realiza tareas de segmentar, síntesis y aislar. Este enfoque 
parece haber recibido un mayor apoyo empírico tal y como se desprende de algunos estudios e informes recientes publicados por comités científicos especializados (Jiménez y Guzmán, 2003; National Reading Panel, 2000). Otra de las variantes del método sintético es el silábico: es parecido a los anteriores, pero en lugar de aprender la letra se aprende la sílaba: "ma" de "mamá", "ta" de "tapa". La mínima unidad de aprendizaje es la sílaba, y no la letra, al menos en los primeros momentos. Una de las desventajas de estos métodos es que son complejos, ya que el niño debe aprender numerosas reglas para articular cada sílaba, y ello trae consigo numerosos errores.

Los métodos globales parten de unidades mayores y concretas, como las frases o las palabras, para llegar luego a las unidades más pequeñas y abstractas (sílabas y letras). Los defensores del enfoque holista en la enseñanza de la lectura (métodos globales o lenguaje integrado) que encuentran su justificación en las teorías del aprendizaje de corte sociocultural, consideran que un objetivo fundamental en la enseñanza de la lectura consiste en plantear esta actividad como un juego de adivinanzas. En general, los métodos globales tienen la ventaja de ser más motivadores al presentar desde el principio la palabra completa o frase con su consiguiente sentido para el lector. Sin embargo, sus detractores comentan que la lectura se hace muy lenta, que son causa de muchos problemas de aprendizaje y que es fácil no percatarse de los pequeños detalles de las letras.

Por último, los métodos mixtos, o llamados también métodos eclécticos (ver para revisión Lebrero y Lebrero, 1996) son aquellos que combinan las ventajas de los métodos sintéticos y analíticos o globales.

Desde la aparición del alfabeto, muchos son los docentes que han preferido enseñar a leer desde las partes al todo, comenzando por la enseñanza de unidades más elementales y aumentando el nivel de complejidad a medida que el niño avanza en la lectura como serían los métodos sintéticos (Jiménez y Hernández, 1986).

Poco a poco, los profesores observaron que este tipo de metodología era muy abstracta y carente de motivación y estimulación por lo que surgieron otros modelos centrados en la enseñanza de unidades más complejas como la palabra o la frase como serían los métodos globales (Alegría, Carrillo y Sánchez, 2005). Sin embargo, estos métodos también presentan sus desventajas, ya que no parecen estar muy justificados cuando los niños aprenden a leer en lenguas que presentan un sistema ortográfico transparente como sería la lengua española, y tampoco serían recomendables para niños disléxicos (Jiménez, 2012).

En nuestro estudio, teniendo en cuenta las teorías del aprendizaje y métodos de enseñanza de la lectura anteriormente expuestos, hemos querido conocer cuáles son las teorías sobre el aprendizaje de la lectura que se atribuye el profesorado de educación infantil y educación primaria que enseñan a leer con diferentes métodos de lectura. 


\section{MÉTODO}

\section{Participantes}

Se contó con una muestra total de 522 profesores de educación infantil y primaria que cumplimentaron un cuestionario atribucional y un cuestionario inicial sobre los métodos de enseñanza de la lectura. Ambos cuestionarios forman parte del programa tutorial LETRA (www.programaletra.ull.es) orientado a la formación del profesorado tutor y de apoyo para la enseñanza de la lectura. La muestra de docentes que dicen utilizar cada uno de los métodos de enseñanza de la lectura identificados se distribuye de la siguiente manera: método mixto $(N=324)$; método global $(N=99)$, método silábico $(N=65)$ y método fonológico $(N=34)$.

\section{Instrumentos}

Cuestionario atribucional. Este cuestionario tiene como objetivo principal averiguar cuáles son las teorías que se atribuyen los profesores acerca del aprendizaje de la lectura. Está compuesto por 60 ítems. Los enunciados que se le presentaban al docente están redactados en términos autorreferenciales. Por ejemplo, "Creo que el niño debe construir su propio aprendizaje de la lectura", "Opino que todos los alumnos necesitan un apoyo del entorno social que complemente el proceso de enseñanza de la lectura”. Los profesores podían expresar su grado de acuerdo o desacuerdo en una escala tipo Likert que va de 0 a 10 , donde 0 significa que los docentes están completamente en desacuerdo con el enunciado y 10 significa que los docentes están completamente de acuerdo con los enunciados presentados. Se elaboró a partir de un estudio representacional previo, donde los enunciados creados fueron valorados por muestras normativas mediante la utilización de "episodios críticos" (para una descripción más detallada del procedimiento, ver Suárez, Jiménez, Guzmán, O’Shanahan y Rodríguez, 2011).

Cuestionario sobre métodos de enseñanza de la lectura. El objetivo es averiguar cuál es el método de lectura que los profesores dicen llevar a cabo en el contexto de clase. Los profesores contestaron a la pregunta ¿Cuál es el método de enseñanza de la lectura que suelen emplear en el aula?, entre las opciones, se encontraban: global (palabra o frase), silábico (fotosilábico o sin imágenes) y fonológico (con o sin onomatopeya). Además, el docente podía elegir más de una opción si lo deseaba.

\section{Procedimiento}

Para realizar el cuestionario atribucional y el cuestionario sobre métodos de lectura, los profesores debían estar inscritos en el programa tutorial de formación para la 
enseñanza de la lectura "LETRA". Cuando recibían el nombre de usuario y contraseña, accedían al sistema tutorial y se les invitaba a cumplimentar los cuestionarios previamente a la formación.

\section{RESULTADOS}

Se analizaron las diferencias entre los cuatro grupos de profesores que dicen seguir diferente metodología para la enseñanza de la lectura en medidas de grado de acuerdo sobre las teorías de aprendizaje. Se consideró el método de enseñanza como factor intersujeto: mixto, global, silábico y fonológico, y como variable dependiente el grado de acuerdo para cada uno de las teorías de aprendizaje que incluye el cuestionario atribucional.

La tabla 1 muestra las medias y desviaciones típicas respecto al grado de acuerdo de las distintas teorías sobre el aprendizaje de la lectura en función del método de enseñanza de la lectura.

Tabla 1. Medias y desviaciones típicas para cada una de las teorías según método de enseñanza

\begin{tabular}{|c|c|c|c|c|}
\hline Teoría & Método & $M$ & $D T$ & $N$ \\
\hline \multirow{4}{*}{ Sociocultural } & $\mathrm{M}$ & .11 & .82 & 324 \\
\hline & $\mathrm{G}$ & -.08 & .92 & 99 \\
\hline & $\mathrm{S}$ & -.25 & 1.01 & 65 \\
\hline & $\mathrm{F}$ & -.37 & 2.04 & 34 \\
\hline \multirow{4}{*}{ Maduracionista } & $\mathrm{M}$ & .04 & .90 & 324 \\
\hline & $\mathrm{G}$ & -.15 & 1.15 & 99 \\
\hline & $S$ & .12 & 1.02 & 65 \\
\hline & $\mathrm{F}$ & .00 & 1.10 & 34 \\
\hline \multirow{4}{*}{ Correctiva } & $\mathrm{M}$ & -.06 & 1.02 & 324 \\
\hline & $\mathrm{G}$ & -.01 & .91 & 99 \\
\hline & $\mathrm{S}$ & .30 & .96 & 65 \\
\hline & $\mathrm{F}$ & .06 & .95 & 34 \\
\hline \multirow{4}{*}{ Repetición } & $\mathrm{M}$ & .02 & .99 & 324 \\
\hline & $\mathrm{G}$ & -.27 & 1.05 & 99 \\
\hline & $\mathrm{S}$ & .32 & .86 & 65 \\
\hline & $\mathrm{F}$ & -.05 & 1.01 & 34 \\
\hline \multirow{4}{*}{ Innatista } & $\mathrm{M}$ & .00 & 1.04 & 324 \\
\hline & $\mathrm{G}$ & -.04 & .82 & 99 \\
\hline & $\mathrm{S}$ & -.01 & .97 & 65 \\
\hline & $\mathrm{F}$ & -.01 & -.97 & 34 \\
\hline \multirow{4}{*}{ Constructivista } & $\mathrm{M}$ & .04 & .93 & 324 \\
\hline & $\mathrm{G}$ & .18 & 1.01 & 99 \\
\hline & $\mathrm{S}$ & -.22 & 1.11 & 65 \\
\hline & $\mathrm{F}$ & -.51 & 1.10 & 34 \\
\hline \multirow{4}{*}{ Psicolingüística } & $\mathrm{M}$ & -.01 & .98 & 324 \\
\hline & $\mathrm{G}$ & -.03 & .96 & 99 \\
\hline & $\mathrm{S}$ & .03 & 1.04 & 65 \\
\hline & $\mathrm{F}$ & .11 & 1.14 & 34 \\
\hline
\end{tabular}

Nota: $\mathrm{M}=$ =étodo mixto; $\mathrm{G}=$ método global; $\mathrm{S}=$ método silábico; $\mathrm{F}=$ método fonológico. 
Se llevó a cabo un modelo lineal general multivariante para analizar si existen diferencias significativas entre las distintas teorías que se atribuyen los profesores sobre el aprendizaje de la lectura y la metodología de enseñanza de la lectura que dicen utilizar y encontramos un efecto significativo $\chi_{(21,1470,73)}=2.86, p<.001 \eta^{2}=.03$. Estas diferencias significativas se encontraron en las teoría sociocultural $F_{(3,518)}=4.77, p<0.001, \eta^{2}=.027$; teoría repetitiva $F_{(3,518)}=5.03, p<0.01, \eta^{2}=.002$ y teoría constructivista $F_{(3,518)}=5.73$, $p<0.001, \eta^{2}=.001$. Cuando analizamos las diferencias par a par (véase tabla 2), encontramos que los profesores que utilizan más el método mixto que el silábico, $t_{(388)}=2.77, p<0.05$ y que el fonológico $t_{(357)}=2.73, p<0.05$, se atribuyen una teoría sociocultural respecto a la enseñanza de la lectura. Por otra parte, los profesores que dicen utilizar más el método silábico, $t_{(33)}=3.80, p<0.001$, que el método global $t_{(224)}=2.66, p<0.05$, se atribuyen una teoría conductista (con mayor énfasis en la repetición). Finalmente, encontramos que los profesores que dicen utilizar más el método global que el fonológico, $t_{(132)}=3.59, p<0.05$, y que el mixto $t_{(357)}=3.18, p<0.05$, piensan que el niño debe construir su propio aprendizaje de la lectura.

Tabla 2. Valores $t$ en las comparaciones múltiples

\begin{tabular}{|c|c|c|c|c|}
\hline \multirow{2}{*}{ Variables dependientes } & \multirow{2}{*}{ Grupo } & \multicolumn{3}{|c|}{$t$} \\
\hline & & $\mathrm{G}$ & $\mathrm{S}$ & $\mathrm{F}$ \\
\hline \multirow{4}{*}{ Maduracionista } & $\mathrm{M}$ & 1.81 & -0.61 & 0.22 \\
\hline & $\mathrm{G}$ & & -1.75 & -0.84 \\
\hline & $\mathrm{S}$ & & & 0.57 \\
\hline & $\mathrm{F}$ & & & \\
\hline \multirow{4}{*}{ Sociocultural } & $\mathrm{M}$ & 1.72 & $2.84 *$ & $2.72 *$ \\
\hline & $\mathrm{G}$ & & 1.13 & -1.45 \\
\hline & $\mathrm{S}$ & & & 0.52 \\
\hline & $\mathrm{F}$ & & & \\
\hline \multirow{4}{*}{ Correctiva } & $\mathrm{M}$ & -0.36 & -2.84 & -0.66 \\
\hline & $\mathrm{G}$ & & -2.00 & -0.4 \\
\hline & $\mathrm{S}$ & & & 1.14 \\
\hline & $\mathrm{F}$ & & & \\
\hline \multirow{4}{*}{ Repetición } & $\mathrm{M}$ & $2.72 *$ & -2.30 & 0.44 \\
\hline & $\mathrm{G}$ & & $-3.75 * * *$ & -1.1 \\
\hline & $\mathrm{S}$ & & & 1.80 \\
\hline & $\mathrm{F}$ & & & \\
\hline \multirow{4}{*}{ Innata } & $\mathrm{M}$ & 0.45 & 0.15 & 0.11 \\
\hline & $\mathrm{G}$ & & 0.125 & -0.15 \\
\hline & $\mathrm{S}$ & & & 0 \\
\hline & $\mathrm{F}$ & & & \\
\hline \multirow{4}{*}{ Constructivista } & $\mathrm{M}$ & -1.27 & 2.15 & $3.16 * *$ \\
\hline & $\mathrm{G}$ & & 2.56 & $-3.5^{* *}$ \\
\hline & $\mathrm{S}$ & & & 1.38 \\
\hline & $\mathrm{F}$ & & & \\
\hline \multirow{4}{*}{ Psicolingüística } & $\mathrm{M}$ & 0.09 & -0.38 & -0.72 \\
\hline & $\mathrm{G}$ & & -0.43 & 0.78 \\
\hline & $S$ & & & -0.38 \\
\hline & $\mathrm{F}$ & & & \\
\hline
\end{tabular}

Nota: $\mathrm{M}=$ =étodo mixto; $\mathrm{G}=$ método global; $\mathrm{S}=$ método silábico; F=método fonológico. $* p<.05 ; * * p<.01 ; * * * p<.001$ 


\section{DISCUSIÓN Y CONCLUSIONES}

El objetivo principal de este estudio ha sido investigar la relación existente entre las teorías sobre el aprendizaje de la lectura que se atribuyen los profesores y el método de enseñanza de la lectura que dicen utilizar en el aula.

Investigaciones previas se han centrado en averiguar qué métodos de lectura utilizan los docentes. Por ejemplo, González, Buisán y Sánchez (2009) encontraron que un $62 \%$ de los profesores encuestados $(N=2.250)$ decía aplicar un método mixto para enseñar a leer, un $16.4 \%$ el método global y un $7.6 \%$ el método fónico, $6 \%$ constructivista, $4.8 \%$ silábico y un $2.7 \%$ otros métodos de lectura. La relación establecida entre los profesores que creen en los postulados de una determinada teoría y los métodos que utilizan en el aula, nos pueden indicar que sus prácticas probablemente se encuentran guiadas por sus propias creencias (Facio, 2003; Stevens, 2002).

Los resultados encontrados en el presente estudio nos muestran que existe relación entre los métodos utilizados por el docente en el aula y las teorías que se atribuyen. Encontramos que los profesores que se atribuyen la teoría sociocultural se inclinan más por la utilización de un método mixto. Los profesores que se atribuyen la teoría conductista, con énfasis en la repetición en los ejercicios y en la instrucción, se inclinan más por utilizar un método silábico y mixto. Por otra parte, los resultados nos indican que los profesores que comparten los principios de la teoría constructivista sobre el aprendizaje utilizan los métodos globales y mixtos para enseñar a leer a sus alumnos.

En este sentido, los profesores que se atribuyen la teoría sociocultural, utilizan los métodos mixtos en el aula, eligiendo las actividades centradas en el sonido, la enseñanza de sílabas, y combinándolo con la enseñanza de palabras y textos, desde una enseñanza explícita en donde el papel que desempeñan los factores culturales y familiares se hace relevante para la enseñanza de la lectura. Los profesores que se atribuyen una teoría del aprendizaje de naturaleza más conductista, basada sobre todo en la repetición, se inclinan más por la utilización de métodos silábicos. Estos concuerdan con sus postulados básicos, ya que inciden en la repetición, memorización y parten de la enseñanza de los elementos más simples de la lectura a los más complejos. Poco a poco, y acorde a su desarrollo se aumenta el nivel de dificultad. Los profesores que se atribuyen postulados de la teoría constructivista, utilizan el método global en el aula centrando sus actividades en lectura de palabras, frases o textos, a través de un proceso interactivo y participativo con el alumno, haciendo que éste construya su propio aprendizaje, un aprendizaje natural y sin necesidad de insistir.

Algunos resultados del presente estudio coinciden con los obtenidos en la investigación llevada a cabo por Clemente, Ramírez y Sánchez (2010). Estos autores analizaron la relación existente entre las tareas declaradas por los docentes y su relación 
con el conocimiento teórico acumulado sobre la enseñanza de la lectura. Encontraron que de los 7 centros participantes en el estudio, las actividades que más correspondencia mostraron con las teorías sobre la enseñanza de la lectura fueron la teoría sociocultural y comunicativa. Además, analizaron la frecuencia de actividades que los docentes decían utilizar en el aula (funciones del lenguaje 11\%, el lenguaje como sistema de representación $22 \%$, el aprendizaje del código $60 \%$ y comprensión lectora $7 \%$ ). Por otro lado, contamos también con otras investigaciones centradas en la alfabetización inicial que han utilizado análisis de caso único (Rodríguez y Clemente, 2013). En esta ocasión estudiaron la relación entre actividades utilizadas por un docente en el aula para enseñar a leer y escribir y su relación con el conocimiento teórico, encontrando que el docente utilizaba actividades relacionadas con las diversas dimensiones, entre ellas la enseñanza del código (p.e., habilidades metalinguiísticas y la enseñanza explícita del código). Además, estos autores encontraron que el docente mantenía una posición integradora de todas las teorías sobre la enseñanza de la lectura.

El reto consiste ahora en averiguar si las teorías que se atribuyen los profesores pueden ser modificadas a través de una formación basada en lo que prescribe la investigación científica sobre cómo enseñar a leer. Un ejemplo de ello lo constituye el Programa LETRA (www.programaletra.ull.es) que ha sido desarrollado por el grupo de investigación DEAP\&NT de la ULL y que ya ha sido pilotado en algunos países del espacio Iberoamericano (por ejemplo, México, Ecuador y Guatemala).

No obstante, una limitación del presente estudio es que al no utilizar metodología observacional no podemos asegurar que las actividades declaradas por los docentes se están implementando en el contexto del aula. Una cosa es lo que piensa y dice que hace el profesor, y otra es lo que realmente hace. Por ello, se requiere investigación, la cual estamos realizando actualmente, donde se intenta integrar el análisis del conjunto de las creencias mediante análisis representacional y atribucional, así como, su relación con las practicas de enseñanza en el contexto del aula mediante metodología observacional.

Agradecimientos: Esta investigación ha sido financiada por el Plan Nacional I+D+i (Ministerio de Economía y Competitividad), con referencia: PSI2009-11662, cuyo IP es Juan E. Jiménez.

\section{REFERENCIAS}

Alegría, J., Carrillo, M. y Sánchez, E. (2005). La enseñanza de la lectura. Revista de Investigación y Ciencia, 340 .

Coltheart, M. (1978). Lexical access in simple reading task. En G. Underwood (Ed.), Strategies of information processing (pp. 151-216). Londres: Academic Press.

Clark, C.M. y Peterson, P.L. (1986). Teachers' thought processes. En M.C. Wittrock (Ed.), Handbook of research on teaching (3rd ed., pp. 255-296). Nueva York: Macmillan. 
Clemente, L., Rodríguez, E. y Sánchez, M.C. (2010). Enfoques teóricos y prácticas docentes en la enseñanza inicial de la lengua escrita. Infancia y Aprendizaje, 22(3), 313-328.

Doman, G.J. (1970). Cómo enseñar a leer a su bebé. Madrid: Aguilar.

Dubois, M.E. (2002). La lectura en la formación y actualización docente. Lectura y Vida, 23(3), 30-38.

Ertmer, A y Newby, J. (1993). Behaviorism, cognitivism: Comparing critical features from and instructional design perspective. Performance Improvement Quartely, 6(4), 50-72.

Fazio, M. (2000). Constructive comprehension and metacognitive strategy instruction in a fieldbased teacher education program. Yearbook of the College Reading Association, 22, 177-190.

Fazio, M. (2003). Constructive comprehension and metacognitive strategy reading instruction in a field-based teacher education program: Effecting change in preservice and inservice teachers participant one. Yearbook of the College Reading Association, 25, 23-45.

González, X.A., Buisán, C. y Sánchez, S. (2009). Las prácticas docentes para enseñar a leer y a escribir. Infancia y Aprendizaje, 32(2), 153-169.

Granda, V. (1998). La Organización de la práctica como una variable de enseñanza aprendizaje. Actas del IV Congreso Nacional de Educación Física de Facultades de Educación y XV de Escuelas Universitarias de Magisterio (pp. 177-206).

Guzmán, R. (1997). Métodos de lectura y acceso al léxico. Tesis doctoral. Universidad de la Laguna.

Hayes, D.A. (1997). Models of professional practice in teacher thinking. En S. Stahk y D.A. Hayes (Eds.), Instructional models in reading (pp. 31-58). Mahwah, NJ: Erlbaum.

Jiménez, J.E. (2012). Dislexia en español. Prevalencia e indicadores cognitivos, culturales, familiares y biológicos. Madrid: Pirámide.

Jiménez, J.E., y Guzmán, R. (2003). The influence of code-oriented versus meaning-oriented approaches to reading instruction on word recognition in a transparent orthography. International Journal of Psychology, 38, 65-78.

Jiménez, J.E. y Hernández, P. (1985). Métodos de lectura y diagnóstico instruccional. Revista de Psicología General y Aplicada, 41, 6, 1063-1074.

Kuzborska, I. (2011). Links between teachers 'beliefs and practices and research on reading. Reading in a Foreign Language, 23(1), 102-128.

Lebrero, M.P. y Lebrero, T. (1996). Cómo y cuándo enseñar a leer y a escribir. Síntesis: Madrid.

Matanzo, J.B. y Harris, D.L. (1999). Encouraging metacognitive awareness in preservice literacy courses. Yearbook of the College Reading Association, 21, 201-225.

McAlpine, L. y Weston, C. (2002). Reflection: Issues related to improving professors' teaching and students' learning. En P. Goodyear y N. Hativa (Eds.), Teacher thinking, beliefs and knowledge in higher education (pp. 59-78). Dordrecht, The Netherlands: Kluwer.

National Reading Panel (2000). Teaching Children to read: An evidence-Based assessment of the scientific research literature on reading and its implications for reading instruction: Reports of the subgroups. Bethesda, MD: National Institute of Child health and Human Development. Avalaible: http://www.nationalreadingpanel.org/.

Pajares, M. (1992). Teachers' Beliefs and Educational Research: Cleaning up a Messy Construct. Review of Educational Research, 62(3), 307-332.

Perkins, D.N. (1991). Technology meets constructivism: Do they make a marriage? Educational Technology, 31(5), 18-23.

Richardson, V. (1996). The role of attitudes and beliefs in learning to teach. En J. Sikula (Ed.), Handbook of research on teacher education (2nd ed., pp. 102-119). Nueva York: Macmillan. 
Rodrigo, M., Rodríguez, A. y Marrero (1993). Las teorías implícitas. Una aproximación al conocimiento cotidiano. Madrid: Alianza Editorial.

Rodríguez, I. y Clemente, L. (2013). Las tareas de enseñanza de la alfabetización inicial en las prácticas docentes. Estudio de caso. Revista Electrónica Interuniversitaria de Formación del Profesorado, 16(1), 41-54.

Sampascual, G. (1985). Evaluación educativa. En J. Mayor, Psicología de la Educación. Madrid: Anaya.

Shavelson, R.J. y Stern, E. (1981). Research on teachers' pedagogical thoughts, judgments, decisions, and behavior. Review of Educational Research, 51, 455-498.

Staub, F. y Stern, E. (2002). The nature of teachers' pedagogical content beliefs matters for students' achievement gains: Quasi-experimental evidence from elementary mathematics. Journal of Educational Psychology, 93, 144-155.

Stevens, L.P. (2002). Making the road by walking: The transition from content area literacy to adolescent literacy. Reading Research and Instruction, 41(3), 267-278.

Suárez, N., Jiménez, J.E., Guzmán, R. y O’Shanahan, J. (2011). Integrando creencias y prácticas sobre enseñanza de la lectura en un sistema tutorial de ayuda asistida por ordenador para la formación del profesorado. Ediciones de la Asociación Nacional de Psicología y de la Educación, 8325-8407.

Theurer, J.L. (2002). The power of retrospective miscue analysis: One preservice teacher's journey as she reconsiders the reading process. Reading Matrix: An International Online Journal, 2(1).

Thompson, A.G. (1992). Teachers' beliefs and conceptions: A synthesis of the research. En D.A. Grouws (Ed.), Handbook of research in mathematics teaching and learning (pp. 127146). Nueva York, NY: Macmillan.

Tracey, D. y Mandel, L. (2012). Lenses on Reading. An introduction to theories and models. Nueva York: The Guilford Press.

Vigotsky, L. (1979). El desarrollo de los procesos psicológicos superiores. Barcelona: Crítica.

Wolf, S.A., Carey, A.A. y Mieras, E.L. (1996a). The art of literary interpretation: Preservice teachers learning about the arts in language arts. National Reading Conference Yearbook, 45, 447-460.

Recibido: 20 de febrero de 2013

Recepción Modificaciones: 8 de marzo de 2013

Aceptado: 7 de enero de 2014 